\title{
HISTOIRE ET AVENIR DE LA PATHOLOGIE FORESTIÈRE EN FRANCE
}

La Pathologie forestière s'intéresse aux maladies de la forêt; au sens le plus communément admis, elle ne concerne que les affections d'origine biotique, provoquées par les microorganismes inférieurs du règne végétal (champignons, bactéries, mycoplasmes, virus). II est clair cependant que les affections d'origine abiotique peuvent interesser également le pathologiste, problèmes carrefour où de nombreuses disciplines peuvent se rencontrer.

Les maladies de la forêt sont l'objet d'un intérêt grandissant de la part d'un public très varié; on parle d'une "montée des pẻrils" et, ce faisant, on ne pense qu'aux événements spectaculaires, vedettes momentanées de la Pathologie forestière. Quelles réalités cela cachet-il ?

Vingt ans après la création du laboratoire de Pathologie forestière du Centre national de Recherches forestières (Institut national de la

C. DELATOUR J. PINON M. MORELET

\section{regards sur la santé de nos forêts} Recherche agronomique), il est temps de regarder le chemin accompli et de préciser les perspectives.

\section{ÉVOLUTION DU CONTEXTE FORESTIER ET CONSÉQUENCES}

L'histoire des maladies des plantes nous montre que les hommes, par leur activitè, ont une grande influence sur la situation phytosanitaire des végétaux qu'ils cultivent. La forêt n'échappe pas à cette règle; aussi n'est-il pas sans intérêt de retracer brièvement les grandes étapes de son évolution dans notre pays pour comprendre les problèmes auxquels est actuellement confrontée la Pathologie forestière. 
L. Badré (1983) distingue trois grandes périodes: "l'Age de la forêt" qui prend fin au début de la Renaissance; "la Pénurie " jusqu'au XIX siècle; puis le "Renouveau " jusqu'à nos jours. Ainsi, après avoir couvert environ 28 millions d'hectares à l'époque romaine, la forêt a longtemps régressé sous l'effet de la pression humaine pour ne plus occuper que 7 millions d'hectares sous le Premier Empire, mais un effort de reboisement sans précédent, entrepris dès le Second Empire, a permis à la forêt de regagner une part importante du terrain perdu pour atteindre actuellement 14 millions d'hectares.

Au cours de ces 2000 ans d'Histoire, le visage de la forêt a également profondément changé.

Aux grandes époques de la régression, c'est le complexe forêt naturelle qui reculait, perdant très probablement une part de sa variété stationnelle; les terrains les plus riches notamment passaient dans le domaine agricole; ce faisant, la forêt elle-même subissait une exploitation grandissante et la baisse de productivité engendrée par la surexploitation contribua au développement de ce qui devint la Sylviculture ; celle-ci, d'abord essentiellement préservatrice de la forêt existante, devint dans les temps modernes plus interventionniste et créatrice. Ses interventions successives nous livrèrent et continuent de modeler les forêts actuelles dont la plupart sont dérivées de la forêt naturelle; ce sont des forêts aménagées par l'homme. Elles constituent un ensemble très varié: les grands massifs résineux des Vosges, du Jura, des Alpes et des Pyrénées, et les grands massifs feuillus de basse altitude dont le principal enjeu est la conversion en futaie, commencée au siècle dernier mais loin d'être achevée. Le passage à la futaie a pour effet de modifier profondément la composition et la structure des peuplements, soit en favorisant des espèces existantes, soit en y introduisant de nouvelles; en outre il a eu longtemps pour objectif l'obtention d'arbres de fortes dimensions, donc âgés.

Enfin, l'intervention la plus récente fut la réalisation de nouvelles forêts qui sont des forêts reconstituées artificiellement. Elles colonisèrent de nouvelles stations, notamment des zones incultes, ou depuis longtemps retournées à l'agriculture; pour ce faire, des procédures variées furent employées : semis ou plantations; interventions culturales plus ou moins intenses (travail du sol, fertilisation, drainage, ligniculture); extension d'espèces autochtones (Pin maritime, Pin sylvestre, Pins noirs, Epicéas...); introduction d'espèces exotiques (Douglas...) ; firent également leur apparition des espèces délibérément sélectionnées, depuis le niveau provenances jusqu'au niveau clonal (Peupliers).

Ainsi, la forêt gauloise est devenue méconnaissable, la forêt naturelle n'existe plus, seules des " forêts aménagées " ou "reconstituées " par l'homme sont actuellement présentes sur notre territoire. Cette évolution, toujours en cours, suit un gradient de productivité et d'artificialisation grandissantes surtout dans les temps récents. Nos forêts modernes sont simplifiées à plusieurs égards, puisqu'elles tendent toutes de façon plus ou moins marquée vers la monospécificité, l'égalité des âges et la réduction de leur base génétique. Cette dérive rappelle celle qui se produit depuis beaucoup plus longtemps dans le domaine agricole et qui nous a enseigné que les pathogènes eux-mêmes suivent l'évolution des formations végétales, s'adaptant continuellement aux nouveaux paysages végétaux que I'homme réalise; ainsi, les équilibres entre la forêt et ses pathogènes évoluent-ils en fonction de l'évolution de la forêt elle-même. Des phénomènes exogènes peuvent en outre venir perturber ce scénario : ce sont en particulier les introductions de pathogènes nouveaux auxquels les végétaux locaux n'ont jamais été confrontés et qui, de ce fait, peuvent subir de graves dommages; l'augmentation moderne en volume et en rapidité des échanges internationaux accroît ce type de risque.

Dans ce contexte général, ce qu'il est seulement possible de dire ici, c'est que la simplification des peuplements forestiers et l'augmentation des risques d'introduction offrent potentiellement aux pathogènes des possibilités nouvelles d'extension, qu'il convient de restreindre au maximum, tout en préservant l'augmentation de la productivité et en respectant les contraintes économiques.

Les brusques ruptures d'équilibre sont des événements qui demeurent très vifs dans les esprits et mobilisent les énergies, comme les grandes épidémies (celles de l'Encre du Châtaignier au siècle dernier, de la Graphiose de l'Orme au cours de ce siècle) ou comme les accidents momentanés (tel le Dépérissement du Chêne en 1976-1980), car ils sont spectaculaires et laissent des traces durables dans 
les peuplements. Les lentes modifications, par contre, ont trop longtemps été négligées, car relativement discrètes en dépit de leur impact économique parfois considérable: Pourriture rouge de l'Epicéa, Chancre du Hêtre, maladies foliaires des.Peupliers en sont des exemples, et c'est le tribut permanent que la forêt paie aux pathogènes.

C'est dans ce contexte que se justifient les actions entreprises et à développer en matière de Pathologie forestière, il convient alors de répondre à trois grandes questions :

- Se préserver des maladies venues d'ailleurs?

Une bonne connaissance des maladies étrangères à notre pays est nécessaire, ainsi qu'un dispositif efficace de législation et de contrôle aux frontières (de nos jours, le service de la Protection des Végétaux se trouve en première ligne dans ce domaine).

- Que se passe-t-il dans nos forêts?

II s'agit ici d'acquérir la meilleure connaissance possible de la situation phytosanitaire française, de prévoir son évolution et de promouvoir les méthodes d'intervention existantes (cette tâche a été dèfinie et organisée pour la première fois en 1972 par la création d'un Dispositif national de Surveillance phytosanitaire de la forêt auquel participent de nombreux organismes).

- En savoir plus et faire mieux?

La réponse à cette question revient pour l'essentiel à la Recherche qui doit contribuer à une meilleure compréhension des phénomènes observés et à l'élaboration de méthodes ou stratégies de parade contre les pathogènes.

\section{ÉVOLUTION DE LA DÉMARCHE SCIENTIFIQUE}

Le développement de la Pathologie forestière dans notre pays est relativement récent: la chronologie historique (tableau I) nous indique quelques événements repères à cet égard. Le premier promoteur de cette discipline est sans contexte d'Arbois de Jubainville, conservateur des Eaux et Forêts, qui fut un ardent propagandiste des premières connaissances acquises en Allemagne dans la deuxième moitié du $X I X^{e}$ siècle. C'est évidemment surtout dans le cadre de l'Ecole des Eaux et Forêts de Nancy que la Pathologie forestière trouva ensuite ses premiers développements (enseignement et recherche) qui demeurèrent cependant assez modestes. II faut attendre le milieu du XXe siècle pour la voir se développer de façon plus importante, puis de façon organisée, favorisée probablement par l'apparition d'un certain nombre d'accidents parasitaires dûment identifiés que l'économie moderne avait de plus en plus de mal à tolérer.

Un pionnier de la Pathologie torestière en France: Alexandre d'Arbois de Jubainville (élève à l'Ecole des Eaux et Forêts, 1855-1857).

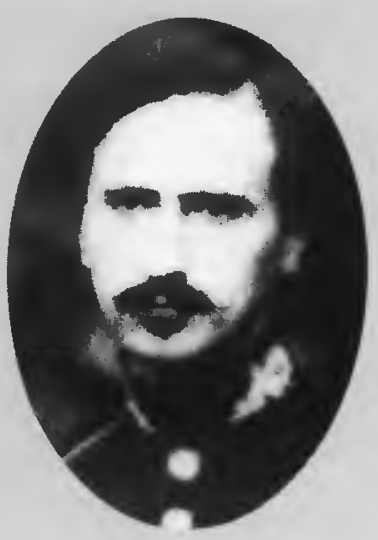


C. DELATOUR - J. PINON - M. MORELET

Tableau ।

Chronologie française pour la Pathologie forestière

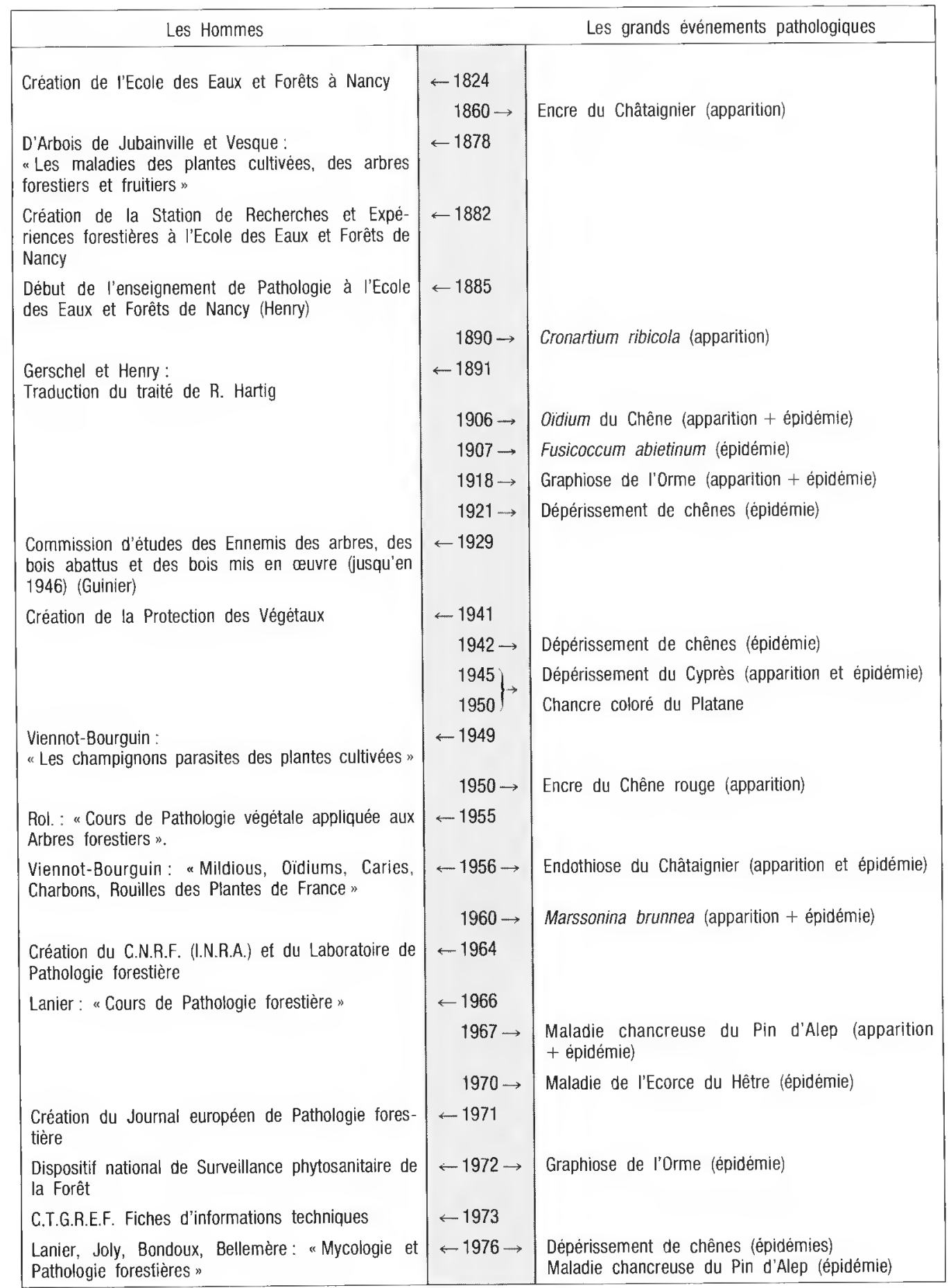


Tableau II Principales mentions originales concernant les maladies forestières en France (jusqu'en 1950)

\begin{tabular}{|c|c|c|c|}
\hline Maladies / Parasites & Auteurs & $\begin{array}{c}\text { Dates de } \\
\text { publication }\end{array}$ & Remarques \\
\hline Hypoderma nervisequum D.C. ............ & De Candolle & 1815 & $1^{*}$ \\
\hline Erysiphe alni D.C. . . . . . . . . & De Candalle & 1815 & 1 \\
\hline Peridermium pineum Chev. . ............... & Chevallier & 1826 & 3 \\
\hline Lophodermium pinastri (Schrad.) Chev. .......... & Chevallier & 1826 & 3 \\
\hline Hymenula platani Lev. . . . . . . . . . . . . . . . . & Léveillé & 1848 & 1 \\
\hline Cladosporium ramulosum Rob. in Desm. ......... & Desmazières & 1852 & 3 \\
\hline Labrella fagi Desm. et Rob. . . . . . . . . . . . . . & Desmazières & 1853 & 1 \\
\hline Nectria ditissima Tul. . . . . . . . . . . . . . & Tulasne & 1865 & 1 \\
\hline Encre du Châtaignier $\ldots \ldots \ldots \ldots \ldots \ldots$ & Planchon & 1878 & 2 \\
\hline Dothichiza populea Sacc. et Br. ............. & Saccardo et Briard & 1884 & 1 \\
\hline Tumeur bactérienne/Pin d'Alep $\ldots \ldots \ldots \ldots \ldots$ & Vuillemin & 1888 & 2 \\
\hline Didymosphaeria populina vuill. . . . . . . . . . . . . & Vuillemin & 1889 & 1 \\
\hline Bacilles de l'Olivier $\ldots \ldots \ldots \ldots \ldots \ldots \ldots \ldots$ & Prillieux & 1890 & 2 \\
\hline Phoma abjetina Hart. . . . . . . . . . . . . . . & Mer & 1890 & 3 \\
\hline Cytospora pinastri Fries $\ldots \ldots \ldots \ldots \ldots \ldots$ & Delacroix & 1892 & 3 \\
\hline Chaudron du Sapin $\ldots . . \ldots \ldots \ldots . . . . .$. & Mer & 1893 & 2 \\
\hline Diplodina castaneae Prill. et Del. ............ & Prillieux et Delacroix & 1893 & 1 \\
\hline Meria laricis Vuill. . . . . . . . . . . . . . . . . & Vuillemin & 1897 & 1 \\
\hline Bandes rouges des pins & Vuillemin & 1897 & 1 \\
\hline Didymascella oxycedri Maire et Sacc. . . . . . . . . . & Maire et Saccardo & 1901 & 1 \\
\hline Rhizosphaera abietis Mang. et Har. . . . . . . . . . . & Mangin et Hariot & 1907 & 3 \\
\hline Pourriture naire des châtaignes $\ldots . \ldots \ldots \ldots \ldots$ & Bainier & 1908 & 2 \\
\hline Oidium alphitoides Grif. et Maubl. . . . . . . . . . . & Griffon et Maublanc & 1910 & 1 \\
\hline Lophodermium macrosporum (Hart.) Rehm ... . . . . & Mer & 1910 & 3 \\
\hline Gymnosporangium tremelloides Hart. ........... & Guinier & 1913 & 3 \\
\hline Rouille vésiculeuse des pins à 5 feuilles & Hatt & 1914 & 2 \\
\hline Maladie de l'Orme ....... & Graffin & 1920 & 2 \\
\hline Sclerotinia betulae Woronin ...... & Malençon & 1924 & 3 \\
\hline Pucciniastrum epilobii (Pers.) Otth. . . . . . . . . . . & Guinier & 1931 & 3 \\
\hline Pucciniastrum goeppertianum (Kühn) Kleb. . . . . . . & Guinier & 1931 & 3 \\
\hline Crumenula pinicola (Reb.) Karst. . . . . . . . . . . . & Guyot & 1934 & 3 \\
\hline Melampsora medusae Thüm. . . . . . . . . . . & Dupias & 1943 & 3 \\
\hline Coryneum cardinale wag. . . . . . . . & Barthelet et Vinot & 1944 & 3 \\
\hline Endothia parasitica (Murr.) And. . . . . . . . . . . & Darpoux & 1948 & 3 \\
\hline
\end{tabular}




\section{DELATOUR - J. PINON - M, MORELET}

Au début, il s'agissait essentiellement de la reconnaissance et de la description des agents pathogènes, mais peu à peu les approches expérimentales et explicatives furent adoptées pour occuper actuellement une place préponderante.

\section{Reconnaissance et description des agents pathogènes}

La fin du XIX siècle et le début du XXe sont caractérisés par le souci de définir les causes des principales affections. Leur citation fournit en même temps des informations sur les problèmes majeurs rencontrès alors en forêt.

Les agents responsables des dégâts sur feuilles ou rameaux sont aisément identifiés. Ils ont souvent été décrits auparavant à l'étranger (tableau II). Dans d'autres cas, la reconnaissance de l'agent causal constitue une première mondiale, ce qui permet aux auteurs de le nommer.

En ce qui concerne les parasites de racines et du collet, la situation est demeurée plus controversée, par exemple pour l'Encre du Châtaignier. Planchon (1878) la nomme ainsi à la suite de ses observations conduites en 1871 en Lozère. II ne croit guère au rôle de l'Armillaire. De Seynes (1879) pense qu'il s'agit d'un champignon nouveau, le Torula exitiosa ${ }^{(1)}$. Pour Cornu (1891), l'Encre serait une conséquence des grands froids, alors que Delacroix (1897) pense que ce sont les agents mycorhiziens qui sont devenus parasites.

Dans le cas de la maladie du Rond, d'Arbois de Jubainville (1875) incrimine Trametes radiciperda et s'oppose vivement en 1878 au baron de Morogues qui, l'année précédente, avait décrit les champignons comme des conséquences et non les causes des dépérissements. En 1879, Seurat de la Boulaye attribue certains d'entre eux à Rhizina inflata, ce que confirmera Duchalais en 1893. Baraban (1881) n'identifie aucun de ces agents bien qu'il croie en l'hypothèse parasitaire.

Dès la fin du XIX ${ }^{e}$ siècle, les études histologiques permettent d'associer, sans pouvoir les identifier, des bactéries à certains symptômes tumoraux, tel le cas du Pin d'Alep étudié par Vuillemin (1888).

De 1914 à 1944, peu de maladies nouvelles sont signalées. Ce fait ne traduit pas nécessairement un meilleur état sanitaire de la forêt, mais plutôt l'indigence des recherches forestières à cette époque. On notera toutefois la conférence de Guinier, en 1930, qui apparaît comme le premier article moderne et synthétique. La période 1944 à 1964 s'enrichit en Mycologie des mentions présentes dans l'ouvrage de Viennot-Bourgin (1949) et marque l'essor de la Bactériologie forestière avec Ridé. De 1964 à nos jours, une trentaine de maladies nouvelles pour la France (et parfois pour le monde) sont découvertes. Ces mentions sont le plus souvent le fait de chercheurs de I'I.N.R.A. et, en particulier, du laboratoire de Nancy, où se développe le premier service de diagnostic forestier. Peu de ces mentions concernent des introductions récentes. Ce sont en fait des lacunes qui sont comblées. Bien que les premiers travaux de Pathologie forestière aient concerné l'Etiologie, celle-ci demeure d'actualité. Elle se précise par l'étude de la variabilité des agents pathogènes: reconnaissance de formes spéciales chez Melampsora allii-populina Niennot-Bourgin, 1937) et Venturia tremulae (Morelet, 1983), de souches chez Ceratocystis ulmi (73-68) ${ }^{(2)}$ et chez Fomes annosus, de races chez Melampsora larici-populina (Pinon et Bachacou, 1984), de spécialisation parasitaire chez l'Armillaire (Guillaumin, 1981) dont l'identification bénéficie des propriétés immunologiques mises en évidence par Lung et Dunez (1980). Un cas original est celui de l'hypovirulence exclusive décrite par Grente et Sauret (1969) chez l'Endothia parasitica.

(1) La nomenclature retenue est celle prẻvalant à l'époque de la publication et non celle en vigeur.

(2) Les références numérotées renvoient à : "Revue analytique des Publications", I.N.R.A.-C.N.R.F. (Unité de Documentation), Champenoux, 54280 SEICHAMPS - FRANCE. 


\section{La Pathologie expérimentale ou explicative}

\section{- L'établissement des cycles parasitaires}

La reconnaissance des agents pathogènes faisait essentiellement appel à ses débuts à l'observation macroscopique des symptômes et des carpophores sur le terrain, complétée assez tôt par celle, microscopique, des organes de fructification. Dès la fin du XIX ${ }^{e}$ siècle, cette approche est complétée par une démarche plus expérimentale impliquant l'inoculation (Cornu, 1878, à propos du Rhytisma acerinum) et/ou la germination provoquée des spores (Henry, 1887, dans le cas de l'Anthracnose du Platane). La maîtrise de ces techniques rend alors possibles les travaux destinés à relier les différentes formes d'un même parasite pour aboutir à la description de son cycle. Ainsi Vuillemin (1887) établit la liaison entre forme sexuée et asexuée dans le cas de la Criblure des Amygdalées, et Prillieux (1889) reconnaît la forme imparfaite du Didymosphaeria populina. Les observations d'Arnaud et de Foex (1912) conduisent à la mise en évidence des périthèces du Blanc du Chêne sessile, ce que confirmera Joessel (1926) sur Chêne pubescent. Arnaud et Barthelet (1933) établissent la liaison entre Phomopsis et Diaporthe oncostoma. Bien que la plupart des liaisons soient à présent établies, une telle démarche demeure actuelle. Ainsi Pinon et Morelet (75-62) ont-ils montré que le Titaeosporina tremulae avait pour forme sexuée le Linospora ceuthocarpa.

La contribution des auteurs français porte aussi sur la reconnaissance des hôtes alternants des Rouilles. Cornu (1880) réussit à contaminer le Dompte-venin à l'aide des écidiospores de Cronartium flaccidum prélevées sur Pin. Il parvient aussi à infecter le Séneçon à l'aide d'écidiospores de Peridermium pini établissant ainsi le cycle de Coleosporium seneciosis. Géneau de Larmalière (1895) montre que l'Aecidium cornui appartient au cycle de C. flaccidum.

A la fin du XIX siècle, peuvent donc débuter les premières études de Pathologie forestière au sens strict, c'est-à-dire celles décrivant les interactions entre l'hôte, son agresseur et le milieu, dans une démarche qui associe l'expérimentation à l'observation, l'analyse à la synthèse. Par simplicité nous conviendrons, dans ce qui suit, d'étudier successivement les trois éléments de ce triangle parasitaire.

\section{- Modes de dissémination et éléments du pouvoir pathogène}

A la fin du $X I X^{e}$ siècle, on ne disposait guère d'appareillages permettant d'étudier la dissémination des spores et l'on devait se résoudre à incriminer pluie et vent. Les observations les plus anciennes sont probablement celles de d'Arbois de Jubainville (1875) à propos de la progression radiale et centrifuge du Fomes. II signale ainsi qu'une mortalité de pins, concernant en 1867 un rayon de sept mètres autour du foyer initial, atteint jusqu'à soixante-dix mètres en 1874. Beauverie (1903) signale que les boutures de Platane sont susceptibles de transmettre l'Anthracnose.

II faut donc attendre la mise au point d'appareils ou de techniques de captures de spores (appareils de Hirst puis de Burkard, rondelles-pièges, gouttières de collecte d'eau) pour que des informations précises soient recueillies en matière de dissémination. Ainsi des liaisons avec la pluie, le vent, la température ont-elles été établies pour les Rouilles du Peuplier (Taris, 1968), le Rouge cryptogamique du Pin (Lanier, 1969), le Marssonina brunnea (85-63), le Fomes (78-110), le Chancre du Hêtre (78-105), celui du Mélèze (81-76) ou la Tavelure des Trembles (Morelet, 1983). Des périodes privilégiées d'émission et de dispersion des spores ont été reconnues pour les parasites foliaires des Peupliers, permettant ainsi de mieux programmer les traitements chimiques en cas de besoin. Mais bien souvent ces émissions s'étendent toute l'année pourvu qu'il pleuve. Ce résultat est trustrant pour les chercheurs, qu'ils aient souhaité définir des périodes optimales pour la lutte chimique ou sylvicole, ou localiser les périodes de contamination pour s'en inspirer lors de leurs inoculations. Cette imprécision a pu être réduite en étudiant les périodes de réceptivité de l'hôte.

Pour la plupart des parasites importants, une certaine variabilité du pouvoir pathogène a été décrite depuis une dizaine d'années. La connaissance des variations de l'agressivité et de la virulence est essentielle, dès lors qu'un programme de sélection pour la résistance est engagé. Nous avons déjà cité 


\section{DELATOUR - J. PINON - M. MORELET}

les exemples pour lesquels des souches, des races ou des formes spéciales sont établies. Dans le cas du Fomes annosus, du Nectria ditissima ou de l'agent du Chancre suintant du Peuplier, des différences entre isolats se sont également fait jour. Les travaux modernes visent à déterminer les armes biochimiques dont disposent les parasites (pectinases, cellulases, ligninases, toxines) et à rechercher les liaisons avec le pouvoir pathogène.

\section{- Le rôle du milieu dans le développement des maladies}

Le milieu peut être scindé en première analyse en deux parties: ses éléments vivants et ses composants abiotiques.

\section{- Microbiologie et Pathologie}

Plusieurs cas d'hyperparasitisme ont été signalés par les auteurs français: Cicinnobolus cesatii sur l'Oïdium du Chêne (Vuillemin, 1910), Fusarium et Tuberculina sur Cronartium ribicola (Lanier, 1969), Darluca filum sur les Rouilles des Salicacées (75-62) et Gonatorrhodiella higlei sur Nectria coccinea (Perrin, 1977). Delatour a étudié en détail la microflore du bois de cœur de l'Epicéa et ses relations (compétition ou inhibition) avec le Fomes annosus. Ridé (1978) a montré que la survie du Xanthomonas populi sur les feuilles de Peuplier était restreinte chez les clones résistants, du fait de l'action antagoniste de la flore associée à ces clones. Ces antagonistes et ces hyperparasites fournissent des éléments pour comprendre la dynamique des populations de parasites, mais ils n'ont jamais encore pu être maîtrisés en vue de la lutte biologique. Des espoirs naissent actuellement du concept de "sols résistants" pour combattre les agents de fonte de semis. Restent enfin à explorer les relations entre les mycorhizes et l'état sanitaire.

\section{- Eléments abiotiques}

Bien que les premiers pathologistes forestiers n'aient pas disposé des méthodes actuelles d'analyse chimique, de biométrie ou de contrôle des facteurs expérimentaux, ils ont pratiqué des observations judicieuses à propos des relations entre la maladie et les caractères stationnels. De Tristan (1894) note la liaison entre le Rhizina et les places à feu, Beauverie (1903) rencontre plus d'Anthracnose sur Platane lorsque le printemps est froid et humide, et Gard (1928) signale l'Armiliaire sur les noyers lorsque ceuxci croissent dans des sols très calcaires. Depuis une quinzaine d'années, les collaborations entre Ecologie et Pathologie se sont multipliées et pour certains parasites les facteurs prédisposants (et donc les stations à risque élevé) ont èté reconnus. Delatour signale ainsi $30 \%$ d'infection des épicéas par le Fomes sur le plateau lorrain contre $15 \%$ dans la plaine. Guillaumin et Delatour ont récemment contribué à étayer l'hypothèse selon laquelle les pathogènes racinaires participent aux grands équilibres de la chênaie. En Normandie, Perrin note que le dépérissement du Hêtre touche plus les stations de plateaux à sol argilo-limoneux que les pentes calcaires. En Lorraine, il rencontre moins de chancres dans les hêtraies calcicoles où la régénération est plus rapide. En Provence, Morelet constate que le Pin d'Alep est particulièrement sensible à la maladie chancreuse lorsqu'il se trouve dans la série thermophile du Chêne vert ou du Chêne pubescent.

Dans les cas précédents, le milieu influençait surtout la résistance de l'hôte. Parfois c'est le parasite qui est concerné et l'effet principal est alors celui du climat. Durrieu (1957), à propos de la Rouille suisse du Douglas, a établi une liaison entre les données climatiques, la disponibilité en ascospores et les possibilités d'infection. Ridé (in Lanier et al, 1976) a montré que le Chancre suintant du Peuplier ne pouvait guère se développer qu'au nord de la Loire. Dupias (1971) a précisé la biogéographie de plusieurs Rouilles, et Somda et Pinon (81-75) ont étudiè expérimentalement leur écophysiologie. Loustau (83-68) a précisé le rôle de la température et des précipitations respectivement sur l'hôte et le parasite dans le cas de la Rouille courbeuse des Pins illustrant ainsi la notion de "résistance phénologique ".

Dans le cas de peuplements artificiels, et en particulier de pépinières, le rôle de la fertilisation comme facteur améliorant ou aggravant l'état sanitaire a été établi expérimentalement. On peut citer ainsi le Rouge cryptogamique du Pin (69-51), le Marssonina brunnea (73-56) et le Chancre du Hêtre (Garbaye et Perrin, sous presse). 
- L'hôte et la maladie

- Ethologie

Dans le précis d'Hartig, il est admis que des conditions particulières sont requises pour l'établissement des relations parasitaires. Si le milieu en module la fréquence et l'importance, comme nous venons de le voir, l'hôte par sa réceptivité et la présence de portes d'entrées pour le parasite joue un rôle déterminant. En 1878, Cornu avait démontré expérimentalement la sensibilité des jeunes feuilles d'Erable à la maladie des croûtes noires. Un siècle plus tard, Cellerino et al. (78-88) établissaient une liaison entre l'âge des feuilles de Peuplier et leur sensibilité à Marssonina brunnea. Des différences importantes de réceptivité ont été définies chez le Peuplier : sensibilité hivernale aux parasites d'écorce (Taris, 1957), pic de sensibilité à l'égard du Chancre suintant lors de la fermeture du bourgeon terminal (Ridé, 1963), contamination plus aisée et plus rapide par Hypoxylon mammatum en période de végétation.

Les voies de pénétration ont souvent èté recherchées. D'Arbois de Jubainville (1880) incriminait les plaies d'élagage dans le cas de Telephora perdrix (= Stereum frustulosum). Selon Dufrenoy (1922), une blessure était nécessaire pour l'installation du Javart sur Châtaignier. Plus récemment, d'autres sites d'infection ont été mis en évidence : cicatrices foliaires - Chancre bactérien (Ridé in Lanier et al., 1976) et Chancre du Pin d'Alep (Morelet) -, lenticelles (Chancre du Hêtre, Perrin), hile des glands (Ciboria batschiana, Delatour et al.) et enfin blessure d'Hylobe (Chancre du Mélèze, Guinot).

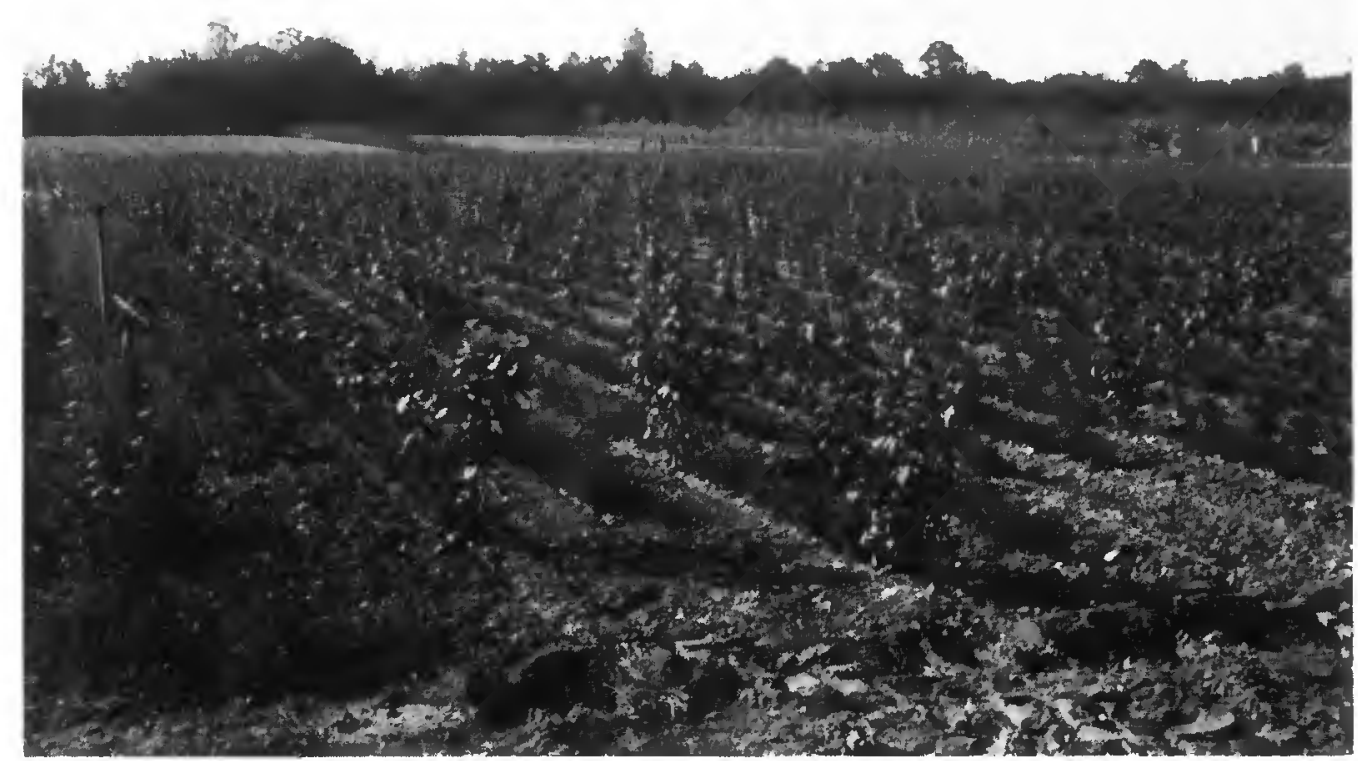

Les tests de sensibilité mettent le matériel végétal en présence d'un Inoculum pathogène homogéne, ce qul est obtenu par introduction dans l'essai d'indivldus particulièrement sensibles que l'on inocule et qui deviennent alors contagieux. Ici, testage de Pins à cinq feuilles pour Cronartium ribicola, les individus contagieux sont ici des Aibes nigrum (hôte alternant). L'implantation multistationnelle permet d'apprécier la dimension écologique de la sensibilité. 


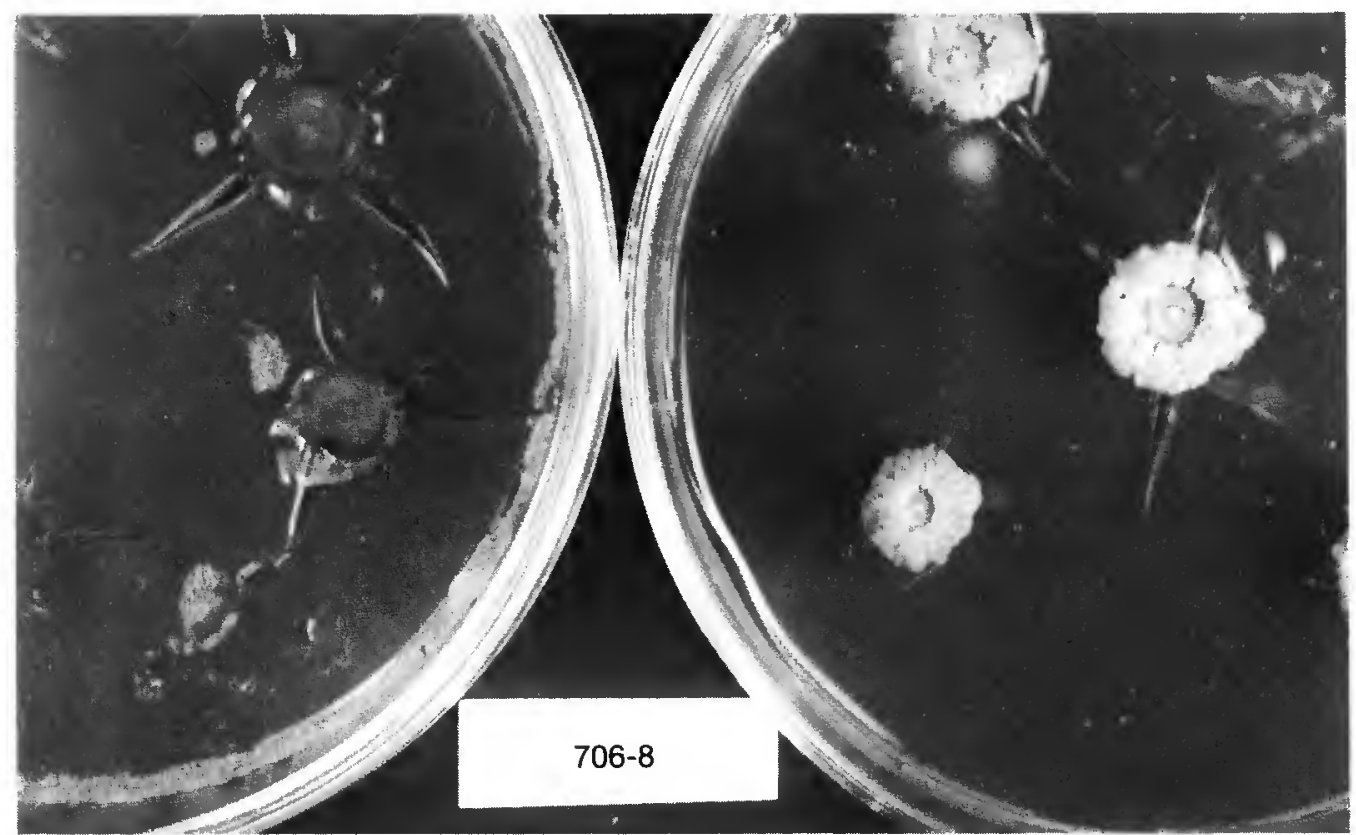

Des tests de laboratolre permettent de recréer certalns aspects de la relation hôte-parasite qul est souvent longue à obtenir en conditions naturelles. Ici, l'effet de la toxine d'Hypoxylon mammatum sur des cals de Populus tremula; ce type d'approche peut permettre de développer des raccourcis méthodologlques Intéressants pour la sélection.

Photo J. PINON

\section{- La variabilité de la résistance de l'hôte}

Pour la plupart des couples hôte-parasite, les variations du degré d'attaque au sein d'une station, ou entre régions, suggèrent une variabilité de la sensibilité de l'hôte. A priori, celle-ci peut refléter aussi bien les effets du milieu que celui des génotypes. A l'égard de l'Armillaire, Morquer et Touvet (1972) ont mis en évidence expérimentalement des différences génériques de résistance à l'Armillaire. Lorsque l'expérimentation en conditions contrôlées conclut au déterminisme génétique de la résistance, il devient possible de sélectionner pour ce caractère (cf. infra).

Afin de résoudre dès que possible les problèmes posés, les pathologistes et les améliorateurs ont privilégié la recherche de sources de résistance. Ce n'est que récemment que l'étude des mécanismes de résistance a été abordée. Chez les pins, Lanier et Hubbes (69-55) ont montré l'activité inhibitrice des extraits de l'hôte à l'égard du Lophodernium pinastri, Loustau (83-68) celle des cires, des exsudats et des terpènes vis-à-vis de Melampsora pinitorqua, et Thibault (82-73) celle des terpènes vis-à-vis du Fomes annosus. Ponchet et Andreoli (1983) ont mis en évidence une réaction histologique de défense (barrière subéreuse) du Cyprès en réponse à l'attaque du Seiridium cardinale (1983). Pinon (1980) a décrit la diversité des composantes phénotypiques de la résistance et abordé les relations entre les produits de la photosynthèse et le parasite.

\section{LE DÉVELOPPEMENT DES MÉTHODES DE LUTTE}

Un peu en amont des problèmes de lutte se trouve l'estimation des dégâts. Malheureusement, il est le plus souvent difficile de chiffrer l'impact des parasites faute de techniques adaptées. Des progrès en ce domaine ont été récemment accomplis. Le premier cas est celui qui concerne des surfaces importantes (un massif forestier) avec parfois la nécessitè de renouveler annuellement les observations. Une étape décisive a été franchie avec l'Unité de Télédétection forestière et en collaboration avec des spécialistes de l'analyse numérique des images de I'I.N.R.A.-Versailles. Ces méthodes ont été appliquées par Perrin 
(1978) au Dépérissement du Hêtre en forêt de Lyons, puis par le groupe I.N.R.A. d'étude du Dépérissement du Chêne, à Tronçais et dans le Sud-Ouest, mais elles ont été utilisées, non pas pour apprécier l'impact général des maladies, mais pour les localiser avec précision sur le terrain et surtout en suivre l'évolution. Le second cas porte sur l'estimation de l'impact sur l'individu avec le souci d'obtenir une information assez précise, de façon rapide et non destructive. Perrin et Delatour (76-71) ont ainsi montré qu'une estimation fiable de la hauteur de pourriture due au Fomes pour l'Epicéa était possible à partir de carottes de sondage. A propos de $M$. brunnea, Joannès et Pinon (82-63) ont amélioré et simplifié la quantification des attaques de feuillage dont l'impact a été évalué en pépinière par l'introduction de témoins protégés chimiquement.

II a ainsi pu être estimé par exemple que le Fomes annosus occasionnerait en France chez le seul Epicéa, des pertes annuelles pouvant dépasser 80 millions de francs, et que les attaques de Marssonina en pépinière peuvent provoquer des pertes de biomasse pouvant atteindre $42 \%$. D'autres dégâts sont moins facilement chiffrables: ce sont par exemple les défauts technologiques engendrés notamment par les agents de chancres. Enfin, l'impact des maladies se traduit également en termes sylvicoles et explique la succession ou la présence de certaines espèces. Le Chêne rouge a été massivement répandu en Pays Basque à la suite du déclin des chênaies autochtones attribué à l'Oidium au début du siècle; la régression du Robusta (production de plants) vers 1958 est due à l'apparition de 1-214 mais a été renforcée par une recrudescence de Dothichiza; 10 ans plus tard, le $1-214$ régressait à son tour à cause de sa sensibilité au Marssonina ; le Dépérissement du Hêtre a conduit au retour du Chêne dans certaine "hêtraies normandes"; la Graphiose de l'Orme a abouti à l'extension des Tilleuls dans nos villes. Certaines espèces très productives sont délaissées: le Pin weymouth pour sa sensibilité au Cronartium, le Mélèze d’Europe pour sa sensibilité en basse altitude à la Pézize.

Outre cette influence des maladies sur le paysage forestier, il existe de nombreuses incidences ou contraintes d'ordre technique car, s'inspirant des progrès accomplis en Pathologie agricole, en particulier à propos de la vigne et des arbres fruitiers, les pathologistes forestiers ont, dès la fin du XIX siècle, recherché des méthodes de lutte. Nous les regrouperons schématiquement en trois catégories.

\section{Lutte sylvicole et prophylaxie}

D'Arbois de Jubainville doit être considéré comme un pionnier dans ce domaine, ce qui lui vaut d'être cité à plusieurs reprises par les traducteurs d'Hartig. II propose ainsi l'élimination des hêtres chancreux (Nectria ditissima) qui devront être remplacés dans les vallons humides et étroits par des résineux. Contre le Fomes, dès 1875, il préconise l'arrachage des souches. II suggère également (1880) l'arrachage des chênes contaminés par Telephora perdrix. Cornu (1878) déduit de ses expériences sur Rhytisma acerinum que la destruction en automne des feuilles infectées pourrait prévenir la maladie. De Tristan (1894) conseille de lutter contre les attaques de Rhizina sur Pin sylvestre en isolant les arbres malades par un fossé. Vuillemin (1889) considère que l'élagage peut réduire les dégâts de Tavelure chez les peupliers noirs. Dans son traité de Sylviculture, Boppe (1889) insiste sur la nécessité d'éliminer les sujets porteurs de carpophores et d'éviter l'élagage chez les essences sensibles aux altèrations.

Les principales règles d'hygiène étaient donc définies dès la fin du $X I X^{\ominus}$ siècle. Les recherches modernes ayant trait à la dissémination et à l'éthologie ont permis de préciser pour quelques couples hôte-parasite ces notions d'hygiène: suppression des préexistants chancreux (Hêtre, 75-55), élagage et enfouissement hivernal des feuilles (Marssonina brunnea, 74-65). L'effet stationnel décrit à propos du Fomes sur l'Epicéa, des Nectria du Hêtre, du Dépérissement du Chêne fournissent des éléments essentiels de la lutte écologique dont l'intérêt est évident et qui continuera à bénéficier des études relatives à l'autécologie des essences. Dans le cas des pépinières et des jeunes plantations, nous avons déjà signalé l'intérêt d'une fertilisation raisonnée qui évite aussi bien les insuffisances que les excès qui ne manquent pas de retentir sur l'état sanitaire. 


\section{Luttes chimique et physique}

Au XIX siècle, la lutte chimique commence à être expérimentée au sein de la Station de Recherches forestières. Le sulfate de cuivre est préconisé par Bartet et Vuillemin (1888) contre le Lophodermium pinastri et par Vuillemin (1889) contre la Tavelure des peupliers noirs. Au début du XXe siècle, Beauverie (1906) conseille la protection des plaies de taille pour s'opposer à l'Anthracnose du Platane, et Delacroix (1906) avancẹ une formule applicable à celle du Peuplier menacé par le Dothichiza. II propose également la désinfection des boutures en les immergeant dans la bouillie bordelaise. Cuif (1911) introduit l'usage du soufre à l'encontre de l'Oídium du Chêne.

Entre les deux guerres, l'accent est mis sur les problèmes sanitaires du Châtaignier: contre la pourriture des châtaignes, Mangin (1918) suggère un tri par flottaison et parvient à les protéger à l'aide de divers désinfectants (sulfure de carbone, naphtol, anhydride sulfureux). Guyot (1936) a expérimenté le sulfate de fer sur Tulipier, l'iode et ses dérivés sur Pin maritime pour lutter contre l'Armillaire dans le Sud-Ouest. Les travaux récents portent essentiellement sur la protection chimique des pépinières et des jeunes peuplements, actuellement seuls justifiables de ces traitements, tant du point de vue technique que du point de vue économique. Les principales mises au point concernant le Rouge cryptogamique du Pin sylvestre (69-52), le Chancre du Hêtre (Perrin, 1977) et le Crumenulopsis sororia du Pin d'Alep. Boudier (1982) a précisé le choix des matières actives opposables à bon nombre de parasites dommageables en pépinière. Un cas particulier est celui de la Graphiose de l'Orme qui a conduit au développement des techniques d'injections et à celui des tests biologiques destinés à tracer les fongicides dans les troncs et les rameaux (80-82, 80-83, 81-70, 82-65).

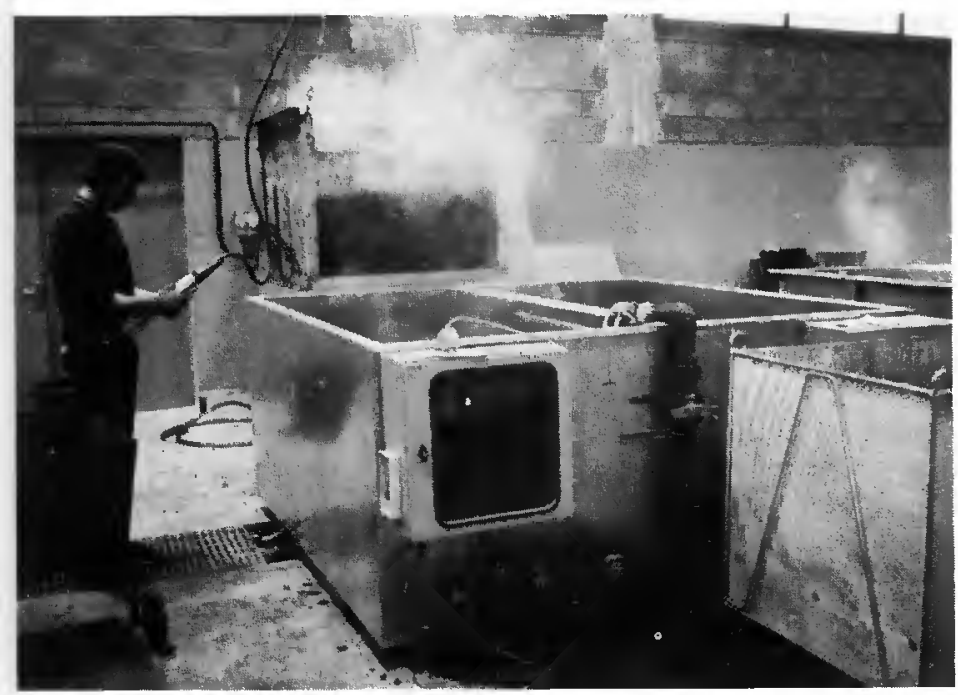

Dans le cas de la pourriture des glands par Ciboria batschiana, Delatour (78-93) a mis au point une méthode de thermothérapie qui détruit le parasite et autorise la conservation prolongée. II en a résulté la création d'unités spécialisées de traitement et stockage des glands au sein de l'Office national des Forêts.

La lutte par thermothérapie contre la pourriture noire a permis d'améliorer sensiblement les perspectives de conservations artificielles des glands. Ici, sortie d'un lot de glands d'une cuve de traitement à la Sécherie de la Joux (O.N.F.)

Photo J.L. RENOUX

\section{Lutte génétique}

Les pratiques forestières usuelles, en éliminant les arbres les plus atteints et en prévenant par conséquent leur multiplication, constituent la forme la plus ancienne de lutte génétique, si on admet qu'il s'agit de résistances gouvernées génétiquement. En 1903, Beauverie suggérait de trier les semis de Platane pour leur résistance à l'Anthracnose. L'Encre du Châtaignier a constitué le souci majeur des pathologistes à la fin du siècle dernier. Dans leur traduction de Hartig, Gerschel et Henry signalent qu'en 1890 la Chambre des députés s'en est émue et a voté des crédits pour l'étude de cette maladie. Les premières tentatives de lutte génétique lui sont consacrées. Dès 1907, Prunet et de Gigord 
signalent l'intérêt des châtaigniers exotiques, ce que confirme Dufrenoy en 1922. Mangin (1925) introduit des châtaignes du Japon et effectue de nombreux semis à Chèvreloup en vue de remplacer l'espèce européenne trop sensible.

La lutte gènétique s'est considérablement développée ces vingt dernières années, la collaboration entre pathologistes et améliorateurs devenant une ligne de force des recherches forestières. Le premier bénéficiaire en fut le Peuplier, sélectionné d'abord pour sa résistance au Chancre suintant, puis pour celle à Marssonina brunnea, aux Rouilles et au Chancre à Hypoxylon. Actuellement, cette sélection est poursuivie activement contre le Chancre du Mélèze et celui du Cyprès. Dans le cas des pins, des essais ont permis d'envisager les possibilités de sélectionner pour la résistance au Lophodermium pinastri, au Cronartium ribicola et à la Rouille courbeuse.

Pour développer des méthodes de test, les pathologistes ont dû consentir un effort important de recherches préliminaires en matière d'éthologie, de dissémination, de conservation d'inoculum, d'expression et de notation des symptômes afin de maîtriser l'infection et d'obtenir des comportements de l'hôte conformes à ceux connus en nature. Parallèlement, on cherche à raccourcir la durée des tests. Ainsi, à propos des parasites foliaires du Peuplier, on vérifie l'intẻrêt des données acquises en pépinière (liaison juvénile/adulte). Dans le cas du Fomes sur Epicéa et de l'Hypoxylon sur Tremble, les recherches s'orientent vers des tests de laboratoire mettant en jeu respectivement "test sciure " et "test toxine". La longueur des cycles de sélection n'a guère permis l'étude de l'héritabilité de la résistance, à l'exception des Rouilles du Peuplier et du Pin maritime.

\section{LA DIFFUSION DES CONNAISSANCES}

\section{La littérature française}

Longtemps la vulgarisation écrite a disposé de moyens limités et dispersés (articles de la Revue des Eaux et Forêts et de la Revue forestière française, de Phytoma, principalement). Un recueil de fiches a éfé publié par le C.T.G.R.E.F. en 1973, suivi d'un second en 1975. De même la littérature scientifique a été dispersée dans les revues françaises. La création du Journal européen de Pathologie forestière par un groupe de chercheurs européens, dont Lanier pour la France, a permis la diffusion des travaux français dans une revue internationale, multilingue et spécialisée.

Les chercheurs ont également tiré profit de l'internationalisation des recherches grâce aux groupes I.U.F.R.O., F.A.O., C.E.E. ou des missions à l'étranger.

Depuis une dizaine d'années, la Pathologie forestière concerne aussi le public qui a pris souvent conscience de son existence par le biais d'essences non strictement forestières (Orme, Platane, Cyprès). Dès lors, les chercheurs ont été sollicités par la presse écrite, la radio et la télévision.

\section{L'enseignement de la Pathologie forestière}

Nous savons peu de choses de l'enseignement de la Pathologie à ses débuts. Lors de la création de l'Ecole forestière de Nancy, cette discipline n'existaif pas et il est probable que les premiers éléments aient èté inclus dans l'enseignement de l'Histoire naturelle (Masson-Four, 1824 à 1826 ; Lamoureux, 1826-1838) puis des Sciences naturelles (Lamoureux, 1838-1880 ; Fliche, 1866-1892 ? ; Henry, 18801885). Vers 1885 , Henry inaugure l'enseignement individualisé de la Pathologie. II sera suivi par Guinier, Rol, Jacamon et enfin par Lanier; actuellement, les chercheurs du laboratoire de Pathologie forestière du C.N.R.F. collaborent également à cet enseignement. 


\section{DELATOUR - J. PINON - M. MORELET}

L'enseignement s'appuya sur des collections d'échantillons. Guyot (1898) signale l'existence à l'Ecole forestière en 1886 "d'un ensemble d'échantillons représentant les difformités et les maladies des arbres", et en 1889, d'Arbois de Jubainville fait don de sa collection mycologique.

De nos jours, la Pathologie forestière figure dans d'autres enseignements que celui de l'Ecole de Nancy, la formation de forestiers de différents niveaux étant assurée par plusieurs établissements; citons simplement à cet égard l'Ecole des Barres (E.N.I.T.E.F.) où un programme officiel de Pathologie existe depuis 1945.

\section{CONCLUSIONS}

Nous avons vu que dans les années récentes, la découverte d'un nombre non négligeable de pathogènes forestiers a eu lieu et il est probable que des lacunes subsistent. De ce fait, l'activité descriptive propre aux périodes de première exploration, peu en vogue de nos jours, demeure encore d'actualité ; elle doit cependant de plus en plus se reconvertir dans ce qui est appelé la "Surveillance". Cette Surveillance, qui a commencé de s'organiser en France il y a une dizaine d'années, bien après celle de nombreux autres pays, doit être une des préoccupations importantes de la forêt française. Elle doit s'intensifier et surtout viser à devenir un outil pertinent: capable de déceler rapidement les anomalies ou évolutions qui surviennent, de juger de leur signification, capable enfin de fournir aux utilisateurs les informations et appuis qui leur sont nécessaires tant en matière de prévention que d'intervention. Ceci ne peut être obtenu que par une coordination améliorée entre les diverses parties prenantes, depuis les propriétaires et gestionnaires jusqu'aux chercheurs spécialisés.

Pour la Recherche proprement dite qui est notre domaine, nous avons déjà eu l'occasion (Delatour, 1984 ; Pinon, 1984) de préciser que les connaissances nouvelles à acquérir relèvent des deux grands domaines complémentaires que sont la résistance d'origine écologique et la résistance d'origine génétique, afin d'être en mesure de contribuer à la constitution de forêts non seulement stables mais encore ne subissant de pertes par les maladies qu'à un niveau compatible avec les exigences économiques; mais beaucoup reste à faire. II est probable que les recherches iront se diversifiant et s'approfondissant, faisant appel plus que par le passé à des méthodes modernes de détection, de physiologie et de biochimie végétales. Ceci nécessitera le recours à une démarche multidisciplinaire accentuée et de haut niveau, sans pour autant perdre la vocation appliquée et préventive.

Si la Pathologie forestière a pu longtemps être considérée, et l'est encore trop souvent, comme une discipline marginale de la foresterie, ceci dans l'avenir sera de moins en moins vrai. Rares sont ceux qui, du chercheur au gestionnaire, de l'enseignant au vulgarisateur, pourront se désintéresser de la "filière phytosanitaire".

$\begin{array}{ccc}\begin{array}{c}\text { C. DELATOUR } \\ \text { Maitre de Recherches }\end{array} & \text { J. PINON } & \text { Maître de Recherches } \\ \text { Laboratoire de Pathologie forestière } & \text { MORELET } \\ \text { Ingénieur }\end{array}$

\section{Remerciements}

$\mathrm{M}^{\text {lle }}$ Lionnet responsable de la Bibliothèque de I'E.N.G.R.E.F/Nancy a facilité grandement la recherche des documents anciens. Qu'elle en soit ici remerciée. 


\section{BIBLIOGRAPHIE}

ANONYME. - Commission d'études des ennemis des arbres, des bois abattus et des bois mis en œeuvre ( 31 bulletins). - Ministère de l'Agriculture. Administration des Eaux et Forêts, 1929-1946.

ANONYME. - Information technique pour la Surveillance et la Protection phytosanitaire de la forêt. - Centre Technique du Génie Rural des Eaux et des Forêts, 1973-1975.

ARNAUD (G.), BARTHELET (J.). - Les chancres du Cedrela et du Robinier. - Revue de Pathologie végétale et d'Entomologie agricole, vol. 20, 1933, pp. 323-332.

ARNAUD (G.), FOEX (E.). - Sur la forme de l'Ö̈dium du Chêne en France. - Comptes rendus hebdomadaires des séances de l'Académie des Sciences, vol. 154, 1912, pp. 124-127.

BADRE (L.). - Histoire de la forêt française. - Paris: Arthaud, 1983. - 310 p.

BAINIER (G.). - Harziella castanae sp. nov. et Stachylidium bicolor. - Bulletin de la Société mycologique de France, vol. 24,1908 , pp. $88-91$.

BARABAN (L.). - Recherches des causes de dépérissernent des pins maritimes dans certaines dunes de la Vendée. Maladie du Rond. - Revue des Eaux et Forêts, vol. 20, 1881, pp. 72-79.

BARIETY (L.), JACQUIOT (C.), MOREAU (C.) et MOREAU (M.). - La maladie de l'Encre du Chêne rouge (Quercus borealis Michx). - Revue de Pathologie végétale et d'Entomologie agricole, vol. 30, 1951, pp. $253-262$.

BARTET (J.), VUILLEMIN (P.). - Recherches sur le rouge des feuilles de Pin sylvestre et sur le traitement à lui appliquer. - Comptes rendus hebdomadaires des séances de l'Académie des Sciences Paris, vol. 106, 1888, pp. 628-630.

BARTHELET (J.), VINOT (M.). - Notes sur les maladies des cultures méridionales. - Annales des Epiphyties, vol. 10 , 1944, pp. $18-20$.

BEAUVERIE (J.). - La maladie des platanes. - Comptes rendus hebdomadaires des seances de l'Académie des Sciences, vol. 136,22 juin 1903, pp. $1586-1588$.

BEAUVERIE (J.). - Sur la maladie des platanes due au Gnomonia veneta (Sacc. et Speg.) Klebahn [Gloeosporium nervisequum (Fuck) Saccardo] particuliërement dans les pépinières. - Comptes rendus hebdomadaires des séances de l'Académie des Sciences, vol. 142, 1906, pp. 1551-1554.

BOPPE (L.). - Traitè de Sylviculture. - Berger-Levrault Editeur, 1889.

BOUDIER (B.). - Intérêt et risques de l'utilisation du Triadiméfon en pépinières forestières et ornementales. - Revue forestière française, vol. $34, \mathrm{n}^{\circ} 6,1982, \mathrm{pp} .399-411$.

CHEVALLIER (F.F.). - Flore générale des environs de Paris 1. - Paris, 1826, p. 385.

CORNU (M.). - Maladie de l'Erable. - Revue des Eaux et Forêts, vol. 17, 1878, pp. 494-495.

CORNU (M.). - Note sur les génèrations alternantes des urédinèes. - Bulletin de la Socièté botanique de France, vol. 27, 1880, pp. 179-183.

CORNU (M.). - Société nationale d'Agriculture (in Hartig, 1891, p. 213).

CUIF (E.). - L'Oidium du Chêne. - Revue des Eaux et Forêts, vol. 50, 1911, p. 270.

D'ARBOIS DE JUBAINVILLE (A.). - Revue des Eaux et Forêts, vol. 3, 1875, p. 105.

D'ARBOIS DE JUBAINVILLE (A.), VESQUE (J.). - Les maladies des plantes cultivées, des arbres fruitiers et forestiers. - Paris : Rotschild, 1878. - 328 p.

D'ARBOIS DE JUBAINVILLE (A.). - Annales de la Société d'Emulation des Vosges, 1880, pp. 1-8.

DE CANDOLLE (A.P.), DE LAMARCKE (J.). - Flore française. - Paris, vol. 6, 1815, p. 104.

DELACROIX (G.). - Contribution à l'étude de la flore mycologique du département de Saône-et-Loire. - Bulletin de la Société d'Histoire naturelle d'Autun, vol. 5, 1892.

DELACROIX (G.). - La maladie des châtaigniers en France, note préliminaire. - Bulletin de la Société mycologique de France, 13, 1897, pp. 242-252.

DELACROIX (G.). - Sur le parasitisme du Dothichiza populea Sacc. et Briard sur diverses espèces de peupliers. Bulletin de la Société mycologique de France, vol. 19, 1903, pp. 353-355.

DELACROIX (G.). - Sur une maladie du Peuplier de la Caroline. - Bulletin de la Société mycologique de France, vol. 22, 1906, pp. 239-253.

DELATOUR (C.), MORELET (M.), MEN (S.). - Le Ciboria batschiana chez les glands: voies de pénétration, évolution en conservation. - Annales de Phytopathologie, vol. 9, 1977, p. 534.

DELATOUR (C.). - Mycoses forestières et résistance écologique. - Comptes rendus hebdomadaires des séances de l'Académie d'Agriculture de France, vol. 70, 1984, pp. 871-880. 


\section{DELATOUR - J. PINON - M. MORELET}

DE SEYNES (J.). - Sur la maladie des châtaigniers. - Comptes rendus hebdomadaires des séances de I'Académie des Sciences, vol. 88, 1879, pp. 36-39.

DESMAZIÉRES (J.B.). - Notices sur les plantes cryptogames de France. - Annales des Sciences naturelles. Botanique, Série 3 , vol. 18,1852, p. 361.

DESMAZIĖRES (J.B.). - Notices sur les plantes cryptogames de France. - Annales des Sciences naturelles. Botanique, Sèrie 3, vol. 20, 1853, p. 225.

DE TRISTAN (?.). - Note sur un cas de maladie ronde dans les massifs de Pin sylvestre. - Annales Comitẻ central agricole de Sologne, 1894, pp. 1-7.

DUCHALAIS (M.). - Rapport sur la maladie du Rond. - Annales Comité central agricole de Sologne, 1893.

DUFRENOY (J.). - La maladie du Châtaignier. - Compte rendu du Congrès règional, Brive, 1922, pp. 45-67.

DUPIAS (G.). - Contribution à l'ètude des Urèdinèes de la Haute-Garonne. - Bulletin de la Socièté d'Histoire naturelle de Toulouse, vol. 78, 1943, pp. 32-52.

DUPIAS (G.). - Essai sur la biogèographie des Urèdinèes. Son apport à la systèmatique. — Bulletin de la Socièté mycologique de France, vol. 87, 1971, pp. 129-243.

DURRIEU (G.). - Influence du climat sur la biologie de Phaeocryptopus gaeumannii parasite du Pseudotsuga. Comptes rendus hebdomadaires des séances de l'Académie des Sciences, vol. 244, 1957, pp. $2183-2185$.

FERRARI (J.P.), PICHENOT (M.). - Ceratocystis fimbriata Ellis et Halsted f. platani (Walter) responsable d'une grave maladie du Platane en France. - Comptes rendus hebdomadaires des séances de l'Académie des Sciences, D, vol. 278, $\mathrm{N}^{\circ} 22,1974$, pp. $2787-2789$.

GARD (M.). - L'Armillaria mellea Vahl. produit deux sortes de pourridiè chez le Noyer. - Revue de Pathologie végétale et d'Entomologie agricole, vol. 13, 1926, pp. 183-185.

GARD (M.). - Pourridiè du Noyer cultivè (Juglans regia) et carbonate de chaux. - Comptes rendus hebdomadaires des seances de l'Acadèmie des Sciences de Paris, vol. 186, $\mathrm{n}^{\circ} 20,1928$, pp. $1373-1375$.

GENEAU DE LAMALIĖRE (L.). - Sur l'ètat écidien du Cronartium flaccidum Wint. - Association française pour l'avancement des Sciences, $23^{e}$ session de Caen, (1894) 1895, pp. 628-629.

GRAFFIN (R.). - Sur la disparition de l'Orme sous les gaz de guerre. - Comptes rendus hebdomadaires de l'Académie d'Agriculture de France, vol. 6, 1920, p. 609.

GRENTE (J.), SAURET (S.). - L'hypovirulence exclusive, phénomène original en Pathologie végétale. - Comptes rendus hebdomadaires des seances de l'Acadėmie des Sciences de Paris, Sèrie D, vol. 268, 1969, pp. $2347-$ 2350 .

GRIFFON (E.), MAUBLANC (A.). - Sur le blanc du Chêne. - Comptes rendus hebdomadaires des séances de l'Acadèmie des Sciences, vol. 147, 1908, pp. 437-439.

GRIFFON (E.), MAUBLANC (A.). - Le blanc du Chêne et l'Oidium quercinum Thümen. - Bulletin de la Société mycologique de France, vol. 26, 1910, pp. 1-6.

GUILLAUMiN (J.J.), BERTHELAY (S.). - Dètermination spècifique des Armillaires par la mèthode des groupes de compatibilitė sexuelle. Spécialisation ècologique des espèces françaises. - Agronomie, vol. 1, 1981, pp. $897-908$.

GUINIER (P.). - Un cas de spécialisation parasitaire chez une Urédinée. Parasitisme de Gymnosporangium treme/loides R. Hart sur l'hybride Sorbus confusa Gremli. - Comptes rendus hebdomadaires des séances de la Sociétẻ de Biologie, vol. 74, 1913, pp. 648-649.

GUINIER (P.). - Les maladies des arbres et les altérations des bois d'origine cryptogamique au point de vue forestier. - Bulletin de la Sociétè des amis et anciens èlèves de l'Ecole nationale des Eaux et Forêts, vol. 9, 1930, pp. 530.

GUINIER (P.). - Note sur deux Pucciniastrum nuisibles aux conifères. Travaux cryptogamiques dẻdiès à L. Mangin. Muséum d'Histoire naturelle de Pans, 1931, pp. 373-375.

GUYOT (C.H.). - L'enseignement forestier en France. - Nancy: Crepin-Leblond, 1898. - 398 p.

GUYOT (A.L.). - Note sur une maladie chancreuse du Pin sylvestre dans le Nord de la France. - Revue de Pathologie végétale et d'Entomologie agricole, vol. 21, 1934, pp. 33-38.

GUYOT (R.). - Traitement rationnel de la maladie du Rond. Congrès de la Noix et de la Châtaigne, Saint-Marcellin (Isère), 1936, $8 \mathrm{p}$.

HARIOT (P.). - Note sur l'Ö̈dium du Chêne. - Bulletin de la Socièté mycologique de France, vol. 23, 1907, pp. 157159.

HARTIG (R.). - Traite des maladies des arbres. (Traduction sur la deuxième èdition allemande par J. GERSCHEL et E. HENRY). - Paris-Nancy: Berger-Levrault Editeur, 1891. - $316 \mathrm{p}$.

HENRY (E.). - La maladie des platanes. - Revue des Eaux et Forêts, vol. 26, 1887, pp. $358-362$. 
JOESSEL (P.H.). - Sur l'abondance des périthèces de Microsphaera quercina (Schw.) Burr. dans la vallée du Rhône au cours de l'année 1926. - Revue de Pathologie végétale et d'Entomologie agricole, vol. 13, 1926, pp. 314-318.

LANIER (L.). - Cours de Pathologie forestière. - Nancy: E.N.G.R.E.F., 1966, 149 p.

LANIER (L.), JOLY (P.), BONDOUX (P.), BELLEMERE (A.). - Mycologie et Pathologie forestière. - Paris: Masson Editeur, 1976. - Tome 2, $478 \mathrm{p}$.

LÉVEILLÉ (J.H.) - Fragments mycologiques. - Annales des Sciences naturelles. Botanique, Série 3, tome 9, 1848, pp. 128.

LUNG-ESCARMANT (B.), DUNEZ (J.). - Les propriétés immunologiques, un critère possible de classification de l'Armillaire. - Annales de Phytopathologie, vol. 12, 1980, pp. 57-70.

MAIRE (R.), SACCARDO (P.A.). - Bulletin de la Société Botanique de France, tome 1, 1901, pp. 205.

MALENÇON (G.). - Le Sclerotinia betulae Woronin. - Bulletin de la Société mycologique de France, vol. 40, 1924, pp. $177-180$.

MANGIN (L.), HARIOT (P.). - Sur la maladie du rouge du Sapin pectiné dans la forêt de la Savine (Jura). - Bulletin de la Société mycologique de France, vol. 23, 1907, pp. 53-68.

MANGIN (L.). - La pourriture des châtaignes. - Comptes rendus hebdomadaires des séances de l'Académie d'Agriculture de France, vol. 4, 1918, pp. 885-889.

MANGIN (L.). - La reconstitution des châtaigneraies détruites par l'Encre. Comptes rendus habdomadaires des séances de l'Académie d'Agriculture de France, vol. 11, 1925, pp. 161-167.

MER (E.). - Description d'une nouvelle maladie des rameaux de Sapin. - Bulletin de la Société botanique de France, vol. 37,1890 , pp. $38-48$.

MER (E.). - Le balai de sorcière du Sapin. Note, Paris, 1893.

MER (E.). - Recherches sur la maladie des branches de Sapin causée par le Phoma abietina R. Hartig. - Journal de Botanique, vol. 7, 1893, pp. 364-375.

MER (E.). - Le Lophodermium macrosporum (Parasite des aiguilles d'Epicéa). Note, 1910.

MER (E.). - Le Lophodermium nervisequum. Note, 1912.

MOREAU (C.), MOREAU (M.). - La " sule " des Sycomores à Paris. - Bulletin de la Sociéte mycologique de France, vol. 67,1952 , pp. 404-418.

MORELET (M.). - Systématique et biologie des Venturia inféodés aux peupliers de la section Leuce. - Thèse, Nancy, $1983,118 \mathrm{p}$.

MORQUER (R.), TOUVET (A.). - Etude expérimentale de la résistance des espèces forestières au parasitisme par Clitocybe mellea. - Comptes rendus hebdomadaires des séances de l'Académie des Sciences de Paris, vol. 274 , 1972, pp. $1038-1043$.

PELHATE (J.), BARBOTIN (F.). - Le dépérissement des thuyas et la maladie des taches foliaires dans l'Ouest, Didymascella thujina. - Phytoma, vol. 104, 1959, pp. 22-24.

PERRIN (R.). - La lutte chimique contre le chancre du Hêtre. - Revue forestière française, vol. 29, 1977, pp. 27-32.

PERRIN (R.). - Gonatorhodiella higlei A.L. Smith. hyperparasite de Nectria coccinea (Pers. ex Fries), un des agents de la maladie du Hêtre. - Comptes rendus hebdomadaires des séances de l'Académie d'Agriculture de France, 1977, pp. $67-70$.

PERRIN (R.). - Résultats préliminaires de l'application de la photographie aérienne infra-rouge couleur à la maladie de l'écorce du hêtre. - Bulletin de la Socièté française de Photogrammétrie, vol. 69, 1978, pp. 16-21.

PINON (J.). - Etude des composants phénotypiques de la résistance à Marssonina brunnea. - Joint Symposium on resistance mechanisms in poplar Diseases, FAO-JUFRO, Kornick, 1-5 september 1980,pp. 141-146.

PINON (J.). - Perspective de la sélection pour la résistance en Pathologie forestière. - Comptes rendus hebdomadaires des séances de l'Académie d'Agriculture de France, vol. 70, 1984, pp. 881-888.

PINON (J.), BACHACOU (J.). - Existence de deux groupes d'isolats differrents par leur pouvoir pathogene chez Melampsora larici-populina Kleb. - Comptes rendus hebdomadaires des séances de l'Académie d'Agriculture de France, vol. 70, 1984, pp. 114-122.

PLANCHON (J.E.). - Maladie des châtaigniers dans les Cévennes. - Revue des Eaux et Forêts, vol. 17, 1878, pp. 554-557.

PONCHET (J.), ANDREOLI (C.). - Relations hôte-parasite dans le couple Cupressus-Coryneum cardinale Wag. Agronomie, vol. 4, 1984, pp. 245-246.

PRILLIEUX (E.). - Sur la maladie du Peuplier pyramidal. - Comptes rendus hebdomadaires des séances de l'Académie des Sciences, vol. 108, 1889, pp. 1133-1 135.

PRILLIEUX (E.). - Les tumeurs à bacilles de l'Olivier comparées à celles du Pin d'Alep. - Comptes rendus hebdomadaires des séances de l'Académie des Sciences, vol. 108, 1889, pp. 249-252. 


\section{DELATOUR - J. PINON - M. MORELET}

PRILLIEUX (E.), DELACROIX (G.). - Le Javart, maladie des châtaigniers. - Bulletin de la Société mycologique de France, vol. 9, 1893, pp. 275-277.

PRILLIEUX (E.). - Maladies des plantes agricoles et des arbres fruitiers et forestiers causées par des parasites végétaux. - Paris : Firmin-Didot, 1895.

PRUNET (A.), DE GIGORD (J.). - Sur la reconstitution des châtaigneraies à l'aide des châtaigniers exotiques. Bulletin de la Sociéte nationale d'Agriculture, 1907.

RIDÉ (M.). - Sur l'étiologie du chancre suintant du Peuplier. - Comptes rendus hebdomadaires des séances de I'Académie des Sciences de Paris, Série D, vol. 246, 1958, pp. $2795-2798$.

RIDÉ (M.). - Our present knowledge of bacterial canker on poplar caused by Aplanobacterium populi. - FAO-CIP, Casale Monferrato, juin 1963.

RIDÉ (M.), RIDÉ (S.), POUTIER (J.C.). - Factor affecting the survival of Xanthomonas populi on aerial structures of poplar. - Proceedings of the IV th international conference on Plant Pathogenic Bacteria, vol. II, 1978, pp. 803814.

ROL (R.). - Cours de Pathologie végétale appliquée aux arbres forestiers. - Nancy: Ecole nationale des Eaux et Forêts, 1955. - 102 p.

ROL (R.). - Le chancre du Chêne rouge d'Amérique. - Revue forestière française, 11, 1951, pp. 704-707.

SACCARDO (P.A.), BRIARD (H.). - Sylloge Fungorum IX, 1884, pp. 672.

SCHNEIDER (A.), MONTANT (C.), LACOSTE (L.). - Taphrina acerina, Exoascée nouvelle pour la France. - Bulletin de la Sociètè d'Histoire naturelle de Toulouse, 93, 1958, pp. 35-38.

SEURAT DE LA BOULAYE (J.). - Mémoire sur la maladie ronde. - Mémoire de la Société d'Agriculture et des Sciences d'Orléans, XXI, 1879, pp. 189-192.

TARIS (B.). - Contribution à l'ètude des maladies cryptogəmiques des rameaux et des jeunes plants de peuplier. Thèse, Alençon, 1957, pp. 282.

TARIS (B.). - Contribution à l'étude des rouilles des Populus observées en France. - Annales des Epiphyties, 19 , 1968, pp. 2-54.

TULASNE (L.). - Selecta fungorum carpologia, 3, Paris, 1865, pp. 73-75.

VIENNOT-BOURGIN (G.). - Contribution à l'étude de la flore cryptogamique du bassin de la Seine (II ${ }^{\ominus}$ note). Deux urédinales nouvelles. - Revue de Pathologie végétale et d'Entomologie agricole, 24, 1937, 75-85.

VIENNOT-BOURGIN (G.). - Les champignons parasites des plantes cultivées. - Paris: Masson Editeur, 1949, pp. 1850.

VIENNOT-BOURGIN (G.). - Mildious, oidiums, caries, charbons, rouilles des plantes de France. - Paris : Lechevalier, 1956, pp. 317.

VUILLEMIN (P.). - Sur une maladie des cerisiers et des pruniers en Lorraine. - Journal de Botanique, 1, 1887, pp. 315-320.

VUILLEMIN (P.). - - Sur les relations des bacilles sur Pin d'Alep avec les tissus vivants. - Comptes rendus hebdomadaires des séances de l'Académie des Sciences, 107, 1888, pp. 1 184-1186.

VUILLEMIN (P.). - La maladie du Peuplier pyramidal. - Revue des Eaux et Forêts, 28, 1889, pp. 266-270.

VUILLEMIN (P.). - Les Hypostomacées, nouvelle famille de champignons parasites. - Bulletin de la Société des Sciences de Nancy, Série II, 14, 1896, pp. 15-67.

VUILLEMIN (P.). - Le déclin de la maladie du blanc du Chêne. - Bulletin de l'Office forestier, 12, 1910, pp. 1-4. 